Down Syndrome Research and Practice Vol. 6, No. 1, pp 43-47

(C) The Down Syndrome Educational Trust

Printed in Great Britain. All Rights Reserved.

ISSN: 0968-7912 (1999) 6:1

\title{
THE WORLD ASSOCIATION MOVEMENT FOR DOWN SYNDROME
}

\author{
Prof. J. Perera \\ Director Prìncipe de Asturias Centre. University of Balearic Islands. (Spain) \\ President of the 6th World Congress on Down syndrome
}

\section{Keys of the World Association movement for Down syndrome}

If we look back at the history of the world association movement for Down syndrome, the impression we have is that during the last decade of the 20th century the aim has been to consolidate the common strength of this independent movement in the world's developed countries, seeking public commitment and collaboration, claiming the representative nature of the collective and organising more solid and better managed structures. The families, professionals and persons with Down syndrome, working together in the associations and federations, try to adapt to the circumstances, to contribute new and specific solutions, to change social attitudes and respond efficiently to the needs of persons with Down syndrome, which is basically the only thing that should really matter to us.

Is it possible for us to talk about a philosophy of our association movement? Do we share a set of ideas and good practices that the majority of the world organisations for persons with Down syndrome assume and accept? Are we clear about the paths we should take as we approach the 21 st century?

To answer these questions, I would like to submit for the consideration of the congress delegates and leaders of the world association movement for Down syndrome, some suggestions and ideas that I have summarised after a close study of the statutes and declarations of more than 40 active associations for Down syndrome from all over the world.

\section{Philosophy of the association movement for Down syndrome: basic principles}

1. The principle that the capabilities of persons with Down syndrome should take precedence over their limitations or differences is assumed from the affirmation of human dignity in which such persons are included.

2. As a result of the Universal Declaration of Human Rights, United Nations' documents and the "World Action Programme for Disabled People", it is affirmed that "the principle of equal rights" between people with and without a disability means that the needs of all individuals have the same importance, that these needs have to form the basis for social planning and that all resources have to be used in such a way that they guarantee equal opportunities for everyone.

3. Our association movement defends the stance that equal rights in health, education, work, social services, culture and leisure are only possible by using ordinary community services, which have to respond to the needs of people with Down syndrome at the same standard as applied to the rest of the population.

4. It is affirmed that personalised and specialised attention in prevention, health, education, employment and similar programmes will tend to cover the specific needs of people with Down syndrome and will be applied by specialists in ordinary centres or services.

5. Normalisation and inclusion at all levels and in all services are defended and only exceptionally, when integration is not possible because of the severity or complexity of the limitations presented by the person with Down syndrome, will that person be attended to in special services or centres, with the family's agreement.

6. It is obvious that special attention is given to the quality of life for people with Down 
syndrome; this being understood as a) that their needs and expectations are covered; $b$ ) that they develop their potentialities; and c) that they enjoy all their rights. Almost all associations refer in one way or another to the term "quality of life".

7. The fight for equality is proposed from the point of view of solidarity, but with respect for diversity in an open and plural society enriched by this diversity and with the active participation of the people with Down syndrome.

8. Association leadership is understood from the point of view of tolerance to be a respectful form of collaborating and participating with all the legally constituted organisations which respect the rights of disabled people, without wishing to resort to competition or confrontation under any circumstances. That is to say, conserving its own identity.

These principles, drawn as I have said from the statutes and declarations of many associations for Down syndrome from all over the world, if placed in logical order, could very well constitute a good universal declaration of the principles of the world association movement for Down syndrome.

However, if principles and ideas are important, so too are good practices. In this case it is much more difficult to try to summarise what is being done in the most active associations in the world because a wide range of variables are involved: standard of development, social habits, customs, ethical principles, government collaboration, etc., etc.

\section{Good practices}

We can, however, try to state some commonly accepted good practices.

Starting from the principle that everyone with Down syndrome has the right to receive the personalised and specialised attention they need, the mission of our associations is to:

- detect these needs

- transmit them to the relevant public organisms - collaborate in solving them; and in two ways:

a) Demanding from the relevant public organisms an efficient and suitable response from the community's ordinary services.

b) Establishing and rendering the necessary services when the public organisms do not have them. In this case:

- Quality services have to be offered.

- They have to be addressed in the same way as companies rendering services to customers (the customer concept implies the right to demand).

- They have to come under the criteria of a non-profitmaking economy (audits, inspection, etc.)

\section{Priorities}

It is not easy either to catalogue our associations' objectives and priorities. Nevertheless, with great respect to what is being done everywhere else and while emphasising not only that there is not one single method, system or way, but also that what works in one place probably does not work elsewhere, I would like to present to you, even though it is in the form of a list, the priorities that our associations establish in their programmes:

In our priorities most emphasis is put on the specificity of the world association movement for the Down syndrome, which at the same time is the greatest difference between our movement and the associations attending to the psychically disabled in general.

a) Prevention. Early diagnosis and genetic advice. It is obvious that good information helps parents to take more and more responsible decisions and to be better prepared to accept their children with Down syndrome.

b) Defence of rights. The associations for Down syndrome will ensure the defence of equal rights recognised by international law for all citizens and will strongly denounce any violations.

c) Specialists' training. The complete training of professionals (physicians, psychologists, educators, speech therapists, trainers for employment, physiotherapists, etc.) is fundamental for people with Down syndrome to obtain specialised medical and educational attention in the community's ordinary services in accordance with their needs.

d) Health programmes. Good health is the basis for intellectual development, as well as for a better quality of life and longer life expectancy. Specific medical problems have to be diagnosed and resolved as soon as possible.

e) Early care programmes. It has been demonstrated that owing to the brain's plasticity during the first years of life early, specific and well applied care programmes are efficient because the children who have followed them improve their cognitive and adaptation capacities.

f) The school of diversity. School has to supply the answer to the educational needs of all its pupils, whether they have a disability or not. No 
effort must be spared to supply schools, public and private ones, with the necessary supports and resources, including those of teacher training, and for curriculum adaptations to be a fact.

g) Support for inclusion in school. Children and young people with Down syndrome who are of school age and are ready to be integrated in ordinary school and yet have not received sufficient specialised support from the relevant education authority will be subject to special attention.

h) Employment. Professional training must prepare for work and for life. The government must give priority to creating jobs in the ordinary labour framework because work means having greater autonomy and being recognised as an adult before the family and before society.

i) Support for the families. From the very beginning the family, as the first natural nucleus of integration for the person with Down syndrome, must have the correct information and proper training, leading to full acceptance of the situation and to effective involvement in the care and education. Likewise, the family must be given support services and rest.

j) Care for the seriously disabled. There are people with Down syndrome who have a profound psychic or physical disability, whose chances of recovery, adaptation to the environment and integration in society are very limited and who constitute a heavy burden for their families. They will receive care, as soon as each case and its special characteristics have been studied, in highly specialised centres, ensuring that the best possible standard of normalisation is achieved in each particular situation.

k) Recognising the role of the adult and selfadvocacy. An education in accordance with the principles of normalisation and integration which is based on the potentialities of the individual leads to a higher level of autonomy and to adopting the adult role. This implies the right to self-advocacy and facilitates integration in work, a relationship with a partner, an independent life and is the best guarantee for facing old age alone.

1) Bringing the services closer to the users. The services must be brought closer to users so that they can improve attention given to the specific needs in each place.

m) Sensitisation, image and promotion. The associations will ensure that a positive image of the person with Down syndrome is promoted in society, from the perspective that diversity enriches the whole.

n) Support for research and the implantation of new technologies. New scientific applications in health care and in the general attention given to the person with Down syndrome will be the object of constant concern so that there is a permanent adaptation of services to scientific standards.

ñ) Guardianship services. By supporting the creation of Guardianship Foundations disabled people are guaranteed to receive correct attention when the family cannot attend to them.

o) Leisure services and free time. To improve the social integration, personal autonomy and enjoyment of recreational activities of people with Down syndrome.

\section{Future prospects}

You are aware of the enormous strength and energy of our associations, but the different challenges we are facing have to be put clearly in focus if we want them to have future prospects. What are these challenges?

1) The first of these could be EFFECTIVENESS. I believe that our associations have to think about achieving results. Good intentions are not enough. We have to achieve what we propose. If our mission is to improve the quality of life of people with Down syndrome, we must evaluate the results. If we propose modifying certain social or political criteria, we have to be effective and achieve maximum collaboration from society.

This approach to effectiveness has to lead to a reassessment of our organisation, our operation and our training. The mission, what we propose to do, is the most important thing, not the entity, nor the persons, nor the professionals, who are only the means, an instrument at the service of a cause.

2) The second challenge is that of SPECIFICITY or SPECIALISATION. Today nobody denies the specificity in the Down syndrome. There are numerous publications all over the world emphasising the unique and particular aspects of Down syndrome which do not occur in other kinds of mental retardation (or which occur in a different proportion) and which therefore mark the limits with other kinds of cerebral pathology. The molecular structure of chromosome 21 shows a series of genetic anomalies which, in turn, cause a series of disorders in the brain and 
in the nervous system for the entire life of people with Down syndrome and which conditions their learning and conduct. The more we know about these specific aspects, the better we will be able to design therapeutic methods and educational strategies that will prove to be more direct and effective for their rehabilitation.

3) The third challenge is a direct consequence of the previous one (that of specificity), and that is the INDEPENDENCE of our associations with respect to associations attending to psychic disabilities in general. Present-day research shows that mental retardation cannot be tackled as one single entity, as it embraces distinct and different symptoms, both from the neuropathological and neuropsychological points of view. The associations of a general nature have not provided the right answer to the needs of people with Down syndrome. That is why families have sought more progressive and up-to-date solutions through the associations for Down syndrome. There is also another important reason and that is the high number in the collective: approximately five million people with Down syndrome in the world. Is that not a sufficient number for the collective to have its own organisation and infrastructure without having to depend on anyone else? Let it be made quite clear - as I have said before - independence does not mean confrontation, neither does it mean denying the many things that are shared in common with the associations working for people with mental retardation nor denying collaboration on certain matters.

4) The fourth challenge is that of COORDINATION OF EFFORT. All too often problems arise in the associations between the parents and professionals, between the associations and centres or services on which these depend, between officials and private interests. Our associations have to make sure that they are above personal interests, professional conflicts, political or religious ideas or any stumbling-block separating them from the only thing that really matters: working to improve the quality of life for people with Down syndrome. One cannot, nor should one, disregard anyone. The coordination of efforts for a common objective will be achieved if parents, professionals and the people with Down syndrome themselves work together, putting aside personal considerations and struggles for power.

5) The fifth challenge is CONSOLIDATION. Our local associations, our regional or national federations and our international confederations need to be stabilised internally in order to acquire the character of democratic change. Too frequently the same persons head an organisation for ten or more years. This is not good. The "personal" work - extraordinary at times - ends with the person. And then comes the disaster. We are all necessary, but no one should think that they are indispensable. The organisations should ensure their stability and their continuity with turns being taken in office: sometimes the parents, at other times the professionals, whoever is better able to do it in each case, and always counting on the actual people with Down syndrome who, more and more, are taking a leading role and want to be their own protectors and defenders.

\section{6) The sixth challenge is COLLABORATION.}

The associations for Down syndrome cannot isolate themselves. The complexity of society today demands the combined intervention of various kinds of entities. While conserving our identity, we have to be capable of seeking and finding spaces for collaboration with other associations who are working with the same aims. We have to find the points that unite us rather than those that separate us. United we can do more and better things. Collaboration and coordination with associations in the sector is - in my opinion - a moral obligation which has to serve to overcome the present mini-nature of the associations. We have to be happy about the triumphs of others, e.g. when someone brings out a new publication, achieves resources for research or attains greater social recognition.

This collaboration has to be extended towards government administration. We should not regard each other as enemies, but as collaborators. We have to ensure that confrontation or servility are not the only means possible for a relationship. We have to take steps towards agreements: our associations cooperate with government administration in providing certain services and in some way we are substituting them by offering services which they should be giving. Probably we do it better and at a lower cost. However, it is necessary to ensure agreements that are over and above political changes and specific individuals.

7) The seventh challenge is that of TRANSPARENCY. If the associations for Down syndrome want the support of people and organisations, administrations, companies and the media, we have to make an effort to be transparent.

Transparent in our ideologies and approaches. Transparent in our message and intentions. Transparent in our organisations, in our 
"dependencies", connections or obligations.

Above all, transparent in the forms of financing and economic management. Transparency is, in this case, synonymous of trust and credibility. We have to avoid the obscure. We have to banish doubt. This is important when public funds are involved, but it is even more so when the funds proceed from solidarity.

8) I would like to give a rather provocative name to the eighth and last challenge:

DISOBEDIENCE. With this term I would like to refer to the need that our associations have to dedicate time, reflection and efforts to constructing what we could call the particular "culture" of our association movement in the world.

I believe that future prospects will be determined - to a great extent - by the capacity we have to get to the heart of those elements that most obviously and clearly identify us. In the theoretical aspects (philosophical-conceptual) as well as in the most practical aspects (strategies, organisation, operation).

Our hallmark is based on the rights that people with Down syndrome have to be treated without any discrimination, in a society that is enriched by diversity. Too frequently we accept charity, welfare, exceptions, when our struggle and our "disobedience" should be claiming constitutional rights that are all too often and in many parts of the world still being denied to persons with Down syndrome.

I know that the challenges I have proposed are difficult. Above all, I know that the challenges in the developing countries are ones of survival and claiming basic rights. However, it seems to me important that we should be clear about the model to follow, the objectives to achieve and that those less advanced societies can count on the unconditional support of the more developed countries.

Let us hope that between us all we are capable of building a better future for people with Down syndrome.

Let us hope that our dreams come true in the 21 st century when we meet at the next World Congress in the year 2000.

\section{Correspondence:}

Prof. J. Perera Director PrÌncipe de Asturias Centre. University of Balearic Islands, Spain (Email: asnimo@lander.es) 